\title{
Saccharide sources do not influence the biofilm formation in Scedosporium/Lomentospora species
}

\author{
Thaís Pereira de Mello ${ }^{1}$, Marta Helena Branquinha ${ }^{2}$ and André Luis Souza dos Santos ${ }^{1,2, *}$ (D) \\ ${ }^{1}$ Laboratório de Estudos Avançados de Microrganismos Emergentes e Resistentes, Departamento de Microbiologia Geral, \\ Instituto de Microbiologia Paulo de Góes, Universidade Federal do Rio de Janeiro, Rio de Janeiro, Brazil, and ${ }^{2}$ Programa de Pós- \\ Graduação em Bioquímica, Instituto de Química, UFRJ, Rio de Janeiro, Brazil \\ ${ }^{\star}$ Corresponding author: Email: andre@micro.ufrj.br
}

(Received 03 October 2019; Revised 13 December 2019; Accepted 14 December 2019)

\begin{abstract}
Scedosporium and Lomentospora species are ubiquitous saprophytic filamentous fungi that emerged as human pathogens with impressive multidrug-resistance profile. The ability to form biofilm over several biotic and abiotic surfaces is one of the characteristics that contributes to their resistance patterns against almost all currently available antifungals. Herein, we have demonstrated that Scedosporium apiospermum, Scedosporium minutisporum, Scedosporium aurantiacum and Lomentospora prolificans were able to form biofilm, in similar amounts, when conidial cells were incubated in a polystyrene substrate containing Sabouraud medium supplemented or not with different concentrations $(2 \%, 5 \%$ and $10 \%)$ of glucose, fructose, sucrose and lactose. Likewise, the glucose supplementation of culture media primarily composed of amino acids (SCFM, synthetic cystic fibrosis medium) and salts (YNB, yeast nitrogen base) did not modulate the biofilm formation of Scedosporium/Lomentospora species. Collectively, the present data reinforce the ability of these opportunistic fungi to colonize and to build biofilm structures under different environmental conditions.
\end{abstract}

Keywords: Scedosporium/Lomentospora; Filamentous fungi; Biofilm; Saccharides; Growth conditions

\section{Introduction}

Scedosporium and Lomentospora species are widely distributed filamentous fungi that emerged as etiologic agents of localized and disseminated diseases in immunocompromised and immunocompetent individuals. Fungal ball and mycetoma are usual clinical manifestations caused by these fungi, resembling a classical biofilm structure (Mello et al., 2019).

Biofilm is considered a microbial community adhered to a biotic/abiotic surface covered by a complex extrapolymeric substance, which confers resistance to environmental stresses (Flemming et al., 2016). Scedosporium and Lomentospora species form biofilm structures over different substrates, including polystyrene, glass, catheters and lung epithelial cells, which present a typical multidrug-resistance profile (Mello et al., 2016, 2018; Rollin-Pinheiro et al., 2017). However, little is known about the environmental conditions that interfere with the biofilm formation in these fungi. In this respect, it is well-established that bacterial/yeast biofilms are influenced by available nutrients, particularly carbohydrates (Jahid et al., 2013; Waldrop et al., 2014; Pereira et al., 2015).

\footnotetext{
(C) The Author(s) 2020. This is an Open Access article, distributed under the terms of the Creative Commons Attribution licence (http:// creativecommons.org/licenses/by/4.0/), which permits unrestricted re-use, distribution, and reproduction in any medium, provided the original work is properly cited.
} 


\section{Objective}

The aim of the present study was to evaluate the influence of monosaccharides (glucose and fructose) and disaccharides (sucrose and lactose) on the ability of S. apiospermum, S. minutisporum, S. aurantiacum and $L$. prolificans conidial cells to form biofilm structures over a polystyrene surface containing different nutritional culture media.

\section{Methods}

Fungi

Scedosporium apiospermum (RKI07_0416) was provided by Dr. Bodo Wanke (Brazil), S. minutisporum (FMR4072), S. aurantiacum (FMR8630) and L. prolificans (FMR3569) were given by Dr. Josep Guarro (Spain). Fungi were maintained in Sabouraud medium for 7 days. Conidia were obtained as previously described (Mello et al. 2016).

\section{Biofilm assay}

Conidia $\left(10^{6}\right.$ cells $)$ were placed to interact $\left(72 \mathrm{~h} / 37^{\circ} \mathrm{C}\right)$ with 96 -well polystyrene plates containing Sabouraud supplemented with different concentrations ( $0 \%, 2 \%, 5 \%$ and $10 \%)$ of glucose, fructose, sucrose and lactose. Synthetic cystic fibrosis medium (SCFM) and yeast nitrogen base (YNB) added or not with $2 \%$ glucose were also tested. Biofilm parameters (biomass, metabolic activity and extracellular matrix) and scanning electron microscopy (SEM) were evaluated as previously published (Mello et al. 2016).

\section{Statistics}

All experiments were performed in triplicate, in three independent experimental sets. Data were expressed as mean \pm standard deviation. Results were evaluated by two-way ANOVA followed by Tukey's test.

\section{Results}

Initially, we analyzed the biofilm formation of Scedosporium/Lomentospora species when cultivated in Sabouraud, a classic culture medium for fungal studies, supplemented or not with different concentrations of monosaccharides (glucose and fructose) and disaccharides (sucrose and lactose). In all fungal species tested, no significant differences were observed regarding the biomass, cellular metabolic activity and production of extracellular matrix comparing the biofilm formed in the absence and presence of soluble carbohydrates, which was also independent of the concentration used (Figs. 1 and 2).

Posteriorly, two additional culture media were selected based on their chemical composition, SCFM (rich in amino acids, which mimics the cystic fibrosis sputum) and YNB (rich in salts), to evaluate the influence of glucose on fungal biofilm formation. Nevertheless, no differences on biofilm biomasses of S. apiospermum, S. minutisporum, S. aurantiacum and L. prolificans were found taking into consideration the media with and without glucose supplementation (Fig. 3).

\section{Discussion}

The ability to adapt to different physicochemical conditions is a central vein in the virulence arsenal of pathogens, because it reflects their ability to colonize several natural environments and anatomic sites of human body. Scedosporium/Lomentospora conidia easily adapt and germinate in a wide range of $\mathrm{pH}$, 


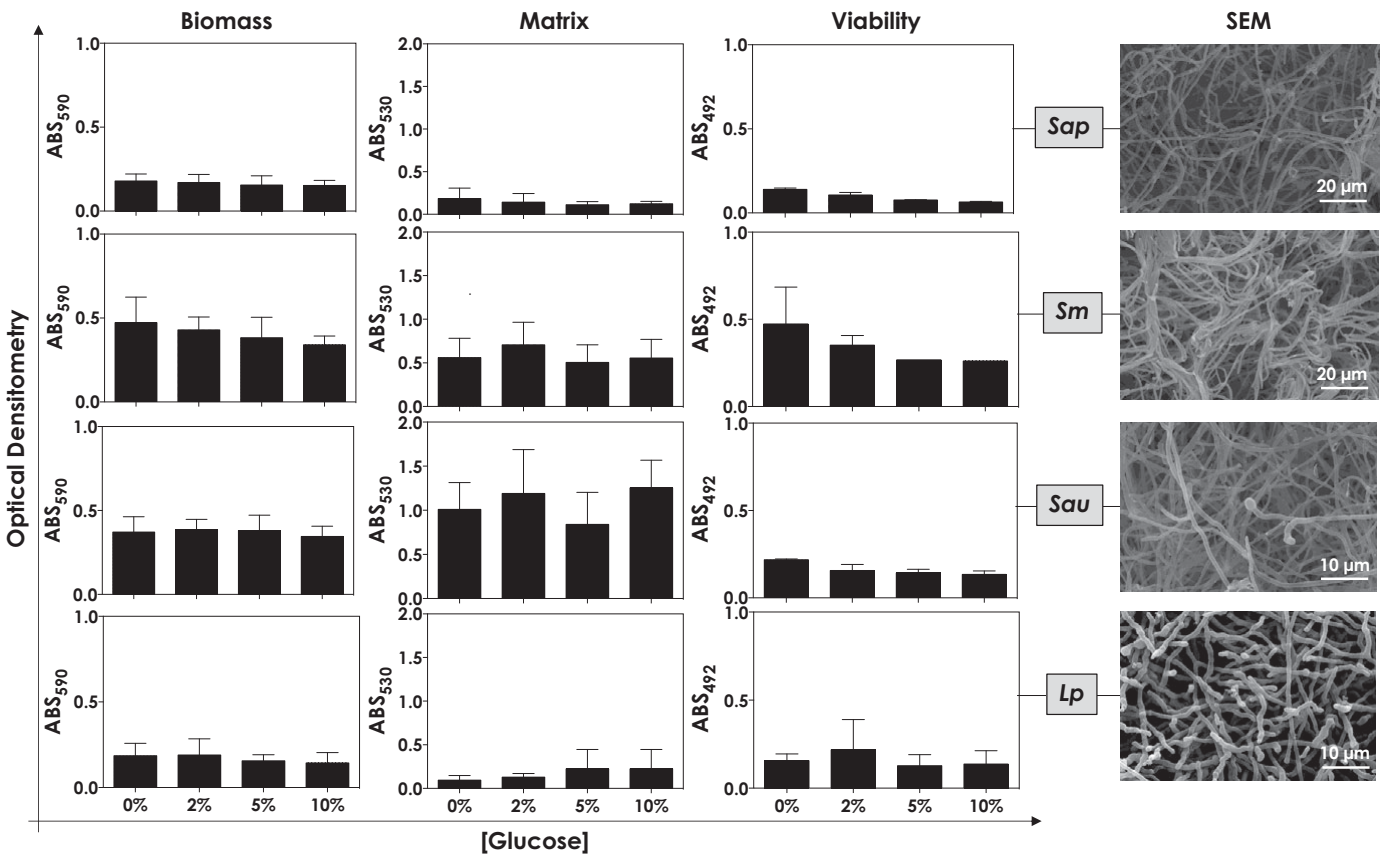

Figure 1. Biofilm formation by S. apiospermum (Sap), S. minutisporum (Sm), S. aurantiacum (Sau) and L. prolificans $(L p)$ under different glucose concentrations. Conidia $\left(10^{6}\right)$ were placed to interact with polystyrene for $72 \mathrm{~h}$ in Sabouraud containing different glucose concentrations $(0 \%, 2 \%, 5 \%$ and $10 \%)$ at $37^{\circ} \mathrm{C}$. Subsequently, in order to assess biofilm formation, the following parameters were spectrophotometrically inspected: biomass was measured in a methanol-fixed biofilm using crystal violet dye $(590 \mathrm{~nm})$ as well as extracellular matrix and metabolic activity (viability) were quantified in non-fixed biofilm by safranin incorporation $(530 \mathrm{~nm})$ and XTT metabolization $(492 \mathrm{~nm})$, respectively. Scanning electron microscopy images of the fungal mature biofilms formed in polystyrene containing Sabouraud supplemented with $2 \%$ of glucose were also shown.
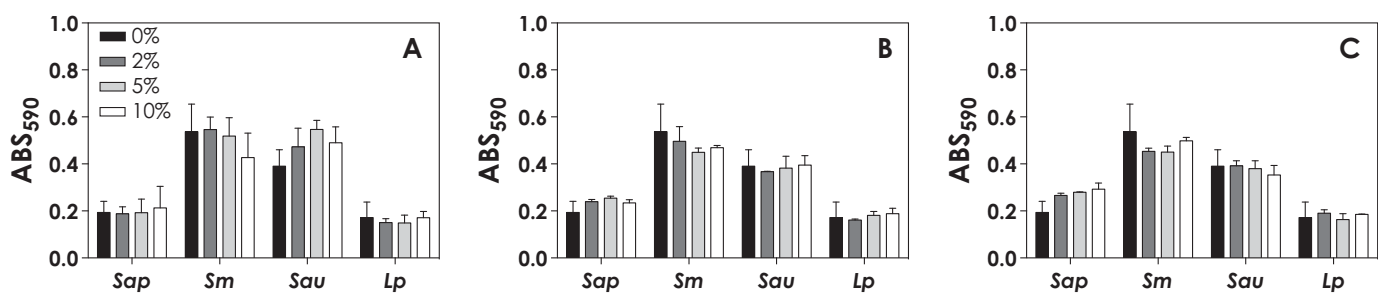

Figure 2. Biofilm formation by S. apiospermum (Sap), S. minutisporum (Sm), S. aurantiacum (Sau) and L. prolificans ( $L p)$ under different saccharide sources. Conidia $\left(10^{6}\right)$ were placed to interact with polystyrene for $72 \mathrm{~h}$ at $37^{\circ} \mathrm{C}$ in $\mathrm{Sabouraud}$ supplemented with different concentrations (0\%, $2 \%, 5 \%$ and $10 \%)$ of fructose (A), sucrose (B) and lactose (C). Then, the systems were processed in order to detect the fungal biomass by incorporation of crystal violet in methanol-fixed biofilms at $590 \mathrm{~nm}$.

temperature and available nutrients (Mello et al. 2016). In addition, these fungal species can adapt to distinct types and concentrations of saccharides, forming a similar amount of biofilm under all tested experimental conditions. Similarly, glucose concentration did not affect the Candida albicans biofilm formation (Kolecka et al.,2015). Conversely, Candida parapsilosis was able to respond to stress caused by glucose (at $10 \%$ ) by up-regulating the genes related to biofilm formation, which culminated to a more robust biofilm formation with a higher concentration of carbohydrates and $\beta$ - $(1,3)$-glucan in the extracellular matrix composition (Pereira et al., 2015). 

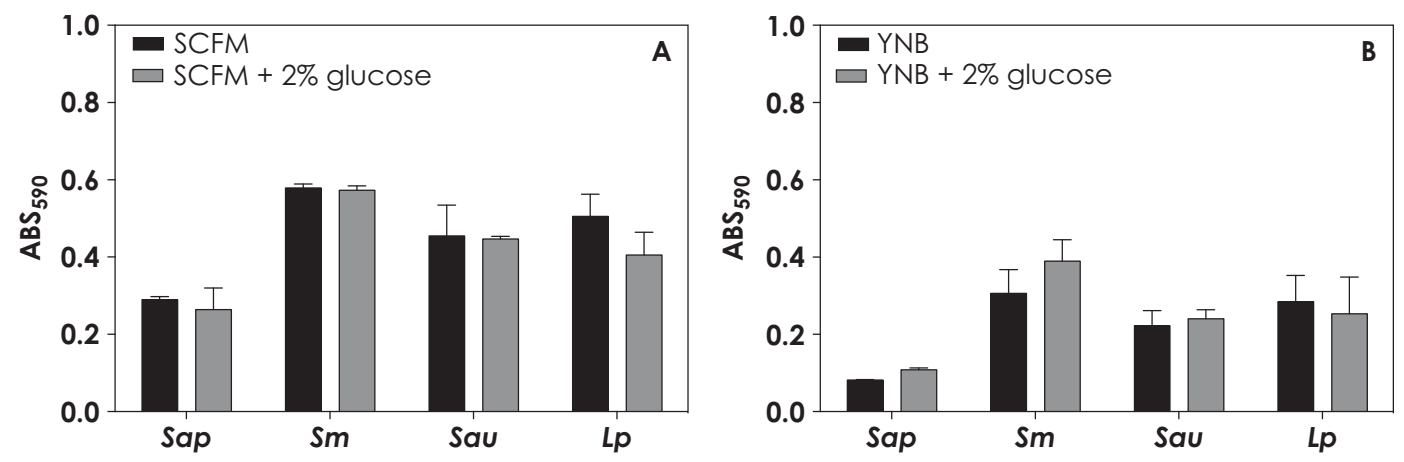

Figure 3. Biofilm formation by S. apiospermum (Sap), S. minutisporum (Sm), S. aurantiacum (Sau) and L. prolificans (Lp) in different culture media. Conidia $\left(10^{6}\right)$ were placed to interact with polystyrene containing SCFM or YNB media supplemented or not with $2 \%$ of glucose for $72 \mathrm{~h}$ at $37^{\circ} \mathrm{C}$. Posteriorly, the systems were processed in order to detect the fungal biomass by incorporation of crystal violet in methanol-fixed biofilms at $590 \mathrm{~nm}$.

\section{Conclusions}

The present findings indicate that soluble carbohydrates (mono- and disaccharides), at different concentrations, were not able to modulate the capacity of $S$. apiospermum, S. minutisporum, S. aurantiacum and L. prolificans to form biofilm on an inert surface containing distinct culture media (Sabouraud, SCFM or YNB). These data can corroborate the high adaptive capacity of these fungi, reflecting in their metabolic plasticity.

Acknowledgments. The authors would like to thank Denise Rocha de Souza and Diego de Souza Gonçalves, both supported by FAPERJ scholarships, for their technical assistance.

Funding Information. This work was supported by grants from Fundação Carlos Chagas Filho de Amparo à Pesquisa do Estado do Rio de Janeiro (FAPERJ), Conselho Nacional de Desenvolvimento Científico e Tecnológico (CNPq) and Coordenação de Aperfeiçoamento de Pessoal de Nível Superior (CAPES - Finance code 001).

Disclosure statement. The authors declare that there is no conflict of interest.

\section{References}

Flemming, H.C., Wingender, J., Szewzyk, U., Steinberg, P., Rice, S.A., Kjelleberg, S. (2016). Biofilms: an emergent form of bacterial life. Nat Rev Microbiol, 14, 563-575.

Jahid, I., Lee, N.Y., Kim, A., \& Ha, S.D. (2013) Influence of glucose concentrations on biofilm formation, motility, exoprotease production, and quorum sensing in Aeromonas hydrophila. J Food Protect, 76, 239-247.

Kolecka, A., Chorvát, D., \& Bujdáková, H. (2015). The impact of growth conditions on biofilm formation and the cell surface hydrophobicity in fluconazole susceptible and tolerant Candida albicans. Folia Microbiol, 60, 45-51.

Mello, T., Bittencourt, V.C.B., Liporagi-Lopes, L.C., Aor, A.C., Branquinha, M.H., \& Santos, A.L.S. (2019). Insights into the social life and obscure side of Scedosporium/Lomentospora species: ubiquitous, emerging and multidrug-resistant opportunistic pathogens. Fungal Biol Rev, 33, 16-46.

Mello, T., Aor, A.C., Gonçalves, D.S., Seabra, S.H., Branquinha, M.H., \& Santos, A.L.S. (2016). Assessment of biofilm formation by Scedosporium apiospermum, S. aurantiacum, S. minutisporum and Lomentospora prolificans. Biofouling, 32, 737-749.

Mello, T., Oliveira, S.S.C., Frasés, S., Branquinha, M.H., \& Santos, A.L.S. (2018). Surface properties, adhesion and biofilm formation on different surfaces by Scedosporium spp. and Lomentospora prolificans. Biofouling, 34, 800-814.

Pereira, L., Silva, S., Ribeiro, B., Henriques, M., \& Azeredo, J. (2015). Influence of glucose concentration on the structure and quantity of biofilms formed by Candida parapsilosis. FEMS Yeast Res, 15, fov043.

Rollin-Pinheiro, R., Meirelles, J.V., Vila, T.V.M., Fonseca, B.B., Alves, V., Frasés, S., Rozental, S., \& Barreto-Bergter, E. (2017). Biofilm formation by Pseudallescheria/Scedosporium species: a comparative study. Front Microbiol, 8, 1568.

Waldrop, R., McLaren, A., Calara, F., \& McLemore, R. (2014). Biofilm growth has a threshold response to glucose in vitro. Clin Orthopaed Rel Res, 472, 3305-3310.

Cite this article: de Mello TP, Branquinha MH, dos Santos ALS (2020). Saccharide sources do not influence the biofilm formation in Scedosporium/Lomentospora species Experimental Results, 1-7. https://doi.org/10.1017/exp.2019.5 


\section{Peer Reviews}

\section{Reviewing editor: Dr. Michael Nevels}

University of St Andrews, Biomolecular Sciences Building, Fife, United Kingdom of Great Britain and Northern Ireland, KY16 9ST

This article has been accepted because it is deemed to be scientifically sound, has the correct controls, has appropriate methodology and is statistically valid, and met required revisions.

doi:10.1017/exp.2019.5.pr1

Review 1: Saccharide sources do not influence the biofilm formation in Scedosporium/ Lomentospora species

Reviewer: Dr. Marcia Pinto

Universidade Federal Fluminense, Microbiologia e parasitologia, Rua Hernani de Melo 101, Niteroi, Brazil, 24220-900

Date of review: 10 October 2019

Published online:

Conflict of interest statement. Reviewer declares none

Comments to the Author: In this study, the authors demonstrated that Scedosporium apiospermum, Scedosporium minutisporum, Scedosporium, aurantiacum and Lomentospora prolificans were able to form biofilm, in similar amounts, when conidial cells were incubated in an abiotic substrate containing Sabouraud medium supplemented or not with different concentrations of glucose, fructose, sucrose and lactose. The problem is significant and it can provide a better understanding of the molecular mechanisms involved in resistance to antifungal agents. The paper presents a good discussion and has scientific merit. The article should be accept.

\section{Score Card}

Presentation

5.0

Is the article written in clear and proper English? (30\%)

Is the data presented in the most useful manner? (40\%)

Does the paper cite relevant and related articles appropriately? (30\%)

Does the title suitably represent the article? (25\%)

Does the abstract correctly embody the content of the article? (25\%)

Does the introduction give appropriate context? (25\%)

Is the objective of the experiment clearly defined? (25\%) 
Are the limitations of the experiment as well as the contributions of the experiment clearly outlined? (20\%) 


\section{Review 2: Saccharide sources do not influence the biofilm formation in Scedosporium/ Lomentospora species}

\section{Reviewer: Dr. Andoni Ramirez-Garcia (D)}

Date of review: 12 December 2019

Published online:

Conflict of interest statement. Reviewer declares none

Comments to the Author: In the manuscript entitled "Saccharide sources do not influence the biofilm formation in Scedosporium/Lomentospora species", the authors demonstrate that S. apiospermum, S. minutisporum, S. aurantiacum and L. prolificans are able to form similar biofilms in Sabouraud, SCFM and YNB supplemented with different concentration of carbohydrates.

Although no differences were found between the different conditions studied, these findings may be interesting as they point out that, in contrast to what have been described for other fungal species, the ability of these fungal pathogens to form biofilms under different nutrients concentration is not altered.

In opinion of this reviewer, only a few details must be modified:

Minor comments:

- Abstract, line 36. Contributes instead of corroborates

- Discussion section should be increased to give more examples to what happen in other species and explain the alterations suffered in biofilm formation under similar variations in environmental conditions (mainly nutrient concentration). In this way, by comparison with the species studied here, the importance of the results found by the authors will be highlighted.

\section{Score Card}

\section{Presentation}

Is the data presented in the most useful manner? (40\%)

Does the paper cite relevant and related articles appropriately? (30\%)

Does the abstract correctly embody the content of the article? (25\%)

Does the introduction give appropriate context? (25\%)

Is the objective of the experiment clearly defined? (25\%)

Are the limitations of the experiment as well as the contributions of the experiment clearly outlined? $(20 \%)$ 